\title{
Call Admission Control for Non-Standalone 5G Ultra-Dense Networks
}

\author{
Saba Al-Rubaye, Senior Member, IEEE, Anwer Al-Dulaimi, Senior Member, IEEE, John Cosmas, \\ Fellow Member, IEEE, and Alagan Anpalagan, Senior Member, IEEE
}

\begin{abstract}
In this letter, we propose new handoff schemes to reduce the interruption time that occurs during re-connection of an arriving mobile user moving from macrocell to small cell or from small cell to macrocell domains. A new call admission control (CAC) function is developed to adjust thresholds during handoff request signaling. To perform the handoff operation, Markov chain technique is used to analyze the call blocking probability characteristic and subsequently to decide handoff approval for various subscriber requests. Numerical results show that the proposed admission control mechanism is able to minimize call blocking probability, without sacrificing resource utilization, and to reduce the number of service interruptions occurring during user re-connections.
\end{abstract}

Index Terms-5G, admission control, handoff scheme, Markov chain model, ultra-dense networks.

\section{INTRODUCTION}

Operators are planning to interface the new radio (NR) $5 \mathrm{G}$ base stations denoted as (gNB) to fourth generation $(4 \mathrm{G})$ core network until migrating to native $5 \mathrm{G}$ core $(5 \mathrm{GC})$ model. This involves interfacing gNBs to $4 \mathrm{G}$ Long TermEvolution (LTA) using (Xx) interface that allows control and user planes access to conventional 4G networking [1]. 5G will be ultra-dense networks (UDNs) and heavily sliced to meet the tremendous increase in data downloading and subscribers connectivity by end-users [2]. Therefore, the time required for processing handoff requests, making handoff decisions, and signalling admission control is very sensitive in term of delay, which required to consider $<1 \mathrm{~ms}$ latency. Since a slice based small cells will be treated as a single network interface by $5 \mathrm{GC}$, managing handoffs between macro-to-small cells and small-to-macro cells is more challenging because of different network interfaces. In this letter, we propose new schemes that improves admission control for macro-tosmall cells and small-to-macro cells using 4G core interfaces to speculate the necessary mechanisms for $5 \mathrm{G}$ developers. The authors in [3] used Markov decision process (MDP) to model admission control mechanism that processes handoff requests provided with prior predictions of requested quality of experience (QoE). The optimization algorithm analyzes individual states to make the necessary approval or rejection actions considering user equipment (UE) traffic and user stratification characteristics. However, most of the (handoff methods) in the literature study signalling schemes without considering admission control constraints. In [4], the authors used 2-D Markov chain to determine call blocking probability and resource availability of UEs at cell edge. This solution is restricted to UE maintaining dual-connectivity with other cooperative macrocells in addition to signal-to-noise ratio (SNR) status. The proposed CAC facilitates call assignment considering different classes of UE speed when re-connecting arrival user from small-to-macro cells or from macro-to-small cells. The handoff requests are assessed based on resource availability and speed where admissions may be blocked in favour of maintaining connectivity over reserved channels that meet quality of service $(\mathrm{QoS})$ requirements. Therefore, the handoff blockage constraint becomes the most important metric in our model since our focus is to derive the resource availability prior to handoff decisions. This reduction in handoff events will also improve stability of resource reservations for sliced networks where spectrum is highly valuable and locally assigned [5]. The objective of the scheme is to meet QoS requirements for any user moving between various operator connected small cells in an open space model. The proposed schemes refer to any LTE or gNB as macrocell and assume no impact in $\mathrm{Xx}$ interface.

\section{SYSTEM ARCHITECTURE MODEL}

The proposed CAC decides to approve or decline handoff once a request is received from an arriving mobile user. In this way, the CAC evaluates the resource availability at the UE original cell and the probability for interruption while waiting for re-connection at the destination cell. Typically, we rely on the macrocell as the main anchor that connects all UEs while small cells are the supplementary service providers. However, the proposed Markov chain model considers all transition states that predict valid handoff requests and activate the blocking probability for others without distinguishing cell type. This makes the proposed mechanism universal and implementable for both small and maco cells.

\section{A. Hand-in Procedure: Macro-to-Small Cell}

The handoff from macro to small cell domains is a challenging procedure considering UDNs where several small cells might be located along the route of target destination. The UE scans for the highest received signal strength from surrounding small cells starting in-band procedure. Then, the UE sends a request to the target small cell for connectivity and once approved, target access point will inform UE and macrocell of approval decision. The procedure starts by handoff request sent through the security gateway to the mobility management entity (MME). The MME then activates the CAC mechanism at the small cell to determine the time of interruption that may 


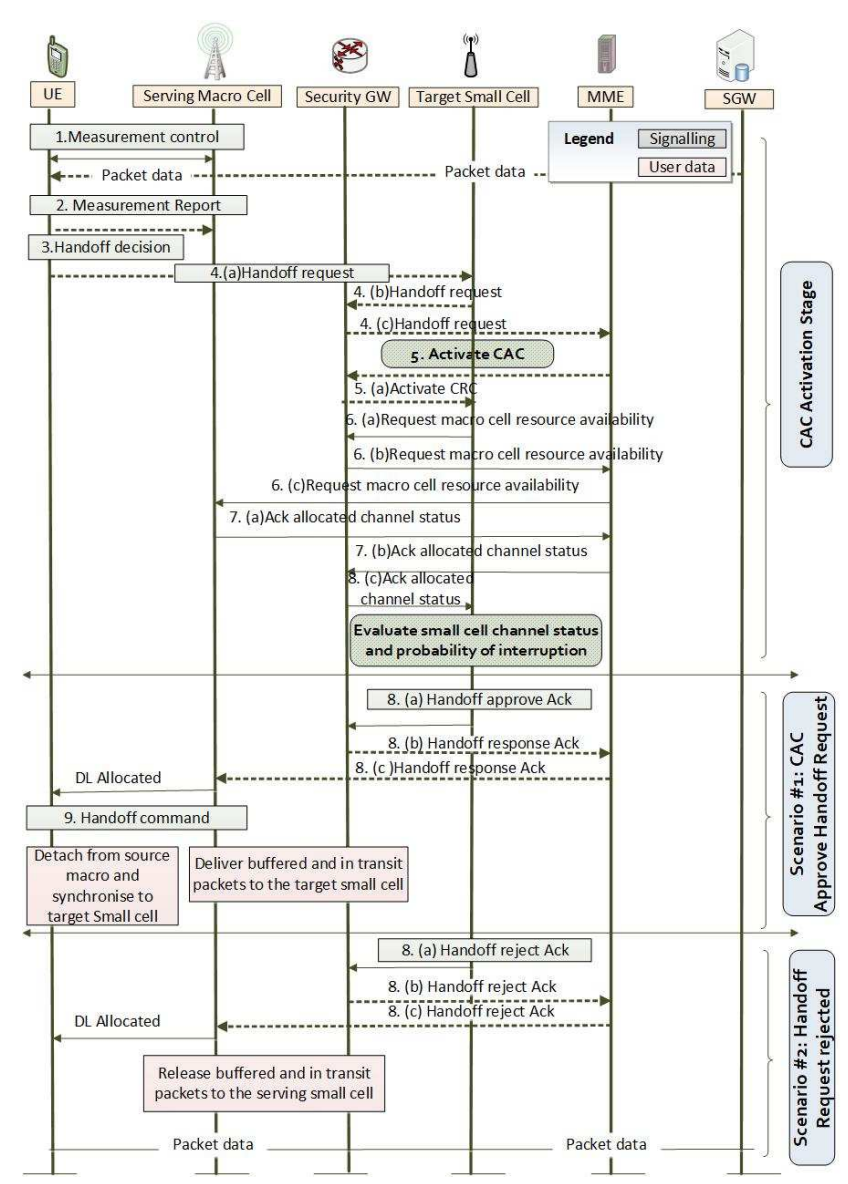

Fig. 1. Handoff from macrocell to small cell.

occur based on channel status at both servicing and target cell. The CAC requests channel status from macrocell and evaluates probability of blocking for admitted UE considering which of the two cells provides more stable connectivity. If handoff request is approved, the data packets are diverted to the target small cell and the link is fully established with the new serving small cell, while UE will be finally disconnected from the macrocell. Using CAC, the small cell computes the resources available to handle the traffic of an arrival UE prior to any handoff decision. If the resources at the small cell is determined to be insufficient to accommodate the arrival UE, the handoff request will be rejected and the MME will issue a request to the macrocell to resume service. The signaling flow diagram for the proposed handoff pattern from macro to small cell is shown in Fig. 1.

\section{B. Hand-out Procedure: Small-to-Macro Cell}

If the UE is moving away from the small cell or just lost connectivity with a certain small cell due to the lack of resources, the UE starts scanning for nearby cells. If no small cell is found, the UE will need to connect to the nearest macrocell determined using the received signal strength (RSS). If the handoff process is initiated, the UE will send a handoff request without any interference or authorization calculations in case of same operator [6]. The handoff request will be

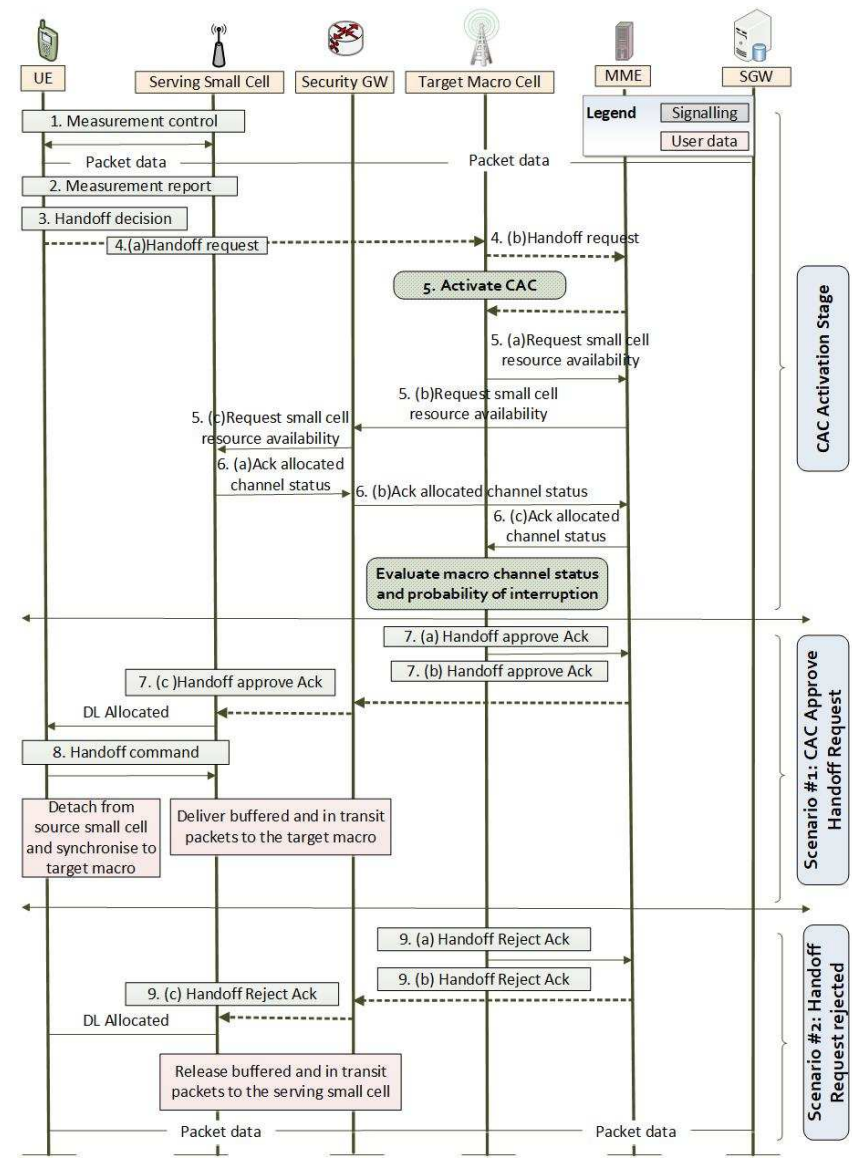

Fig. 2. Handoff from small cell to macrocell.

forwarded by the macrocell to the MME that sends back an activation signal to macro CAC. The CAC will then determine if a suitable channel is available to avoid any interruptions or major delay during the re-registration process. If handoff approved, the serving small cell will re-route undelivered packets via service gateway (SGW) to the target macrocell. In case handoff is request rejected, the serving small cell will be notified to maintain current link, as shown in Fig. 2.

\section{MARKOV CHAIN MODEL FOR HANDOFF DECISIONS}

An efficient handoff policy to improve the resource utilization is achieved by employing CAC technique that adapts to variation of QoS metrics. Markov chain model based queuing analysis is employed to analyze the handoff probability and support adaptive CAC mechanism for both small cell and macrocell domains, as shown in Fig. 3. The occurrence of handoffs for an object state QoS is determined by scaling the steady state probability of that state [7].

In queuing theory, the arrivals of UEs is modelled by applying $M / M / 1$ queue that also represents the queue length for a system determined by Poisson process [8]. In this way, the packet arrival at a given time interval $t$ can be modeled using producer $\lambda t$ and that moves the process from state $i$ to $i+1$. The distribution function of packet service times is exponentially represented using a rate parameter $\mu$ in the 


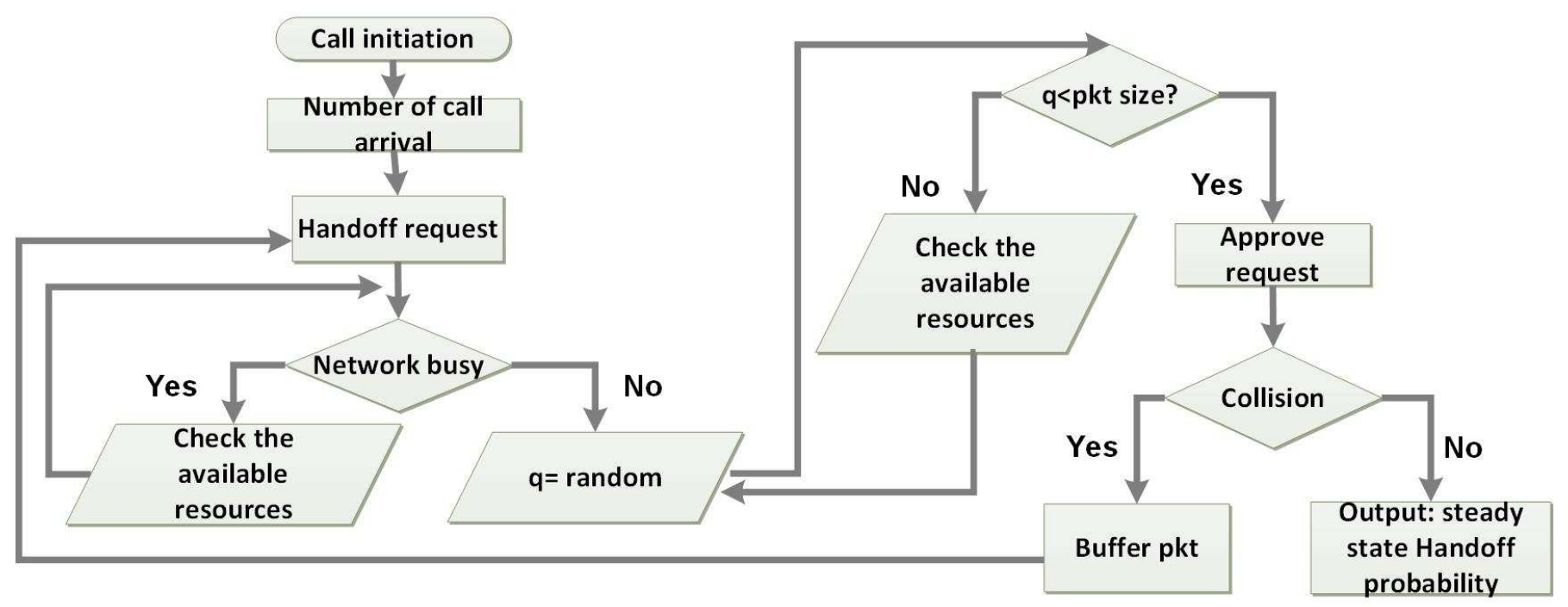

Fig. 3. Markov chain procedure for the proposed CAC scheme.

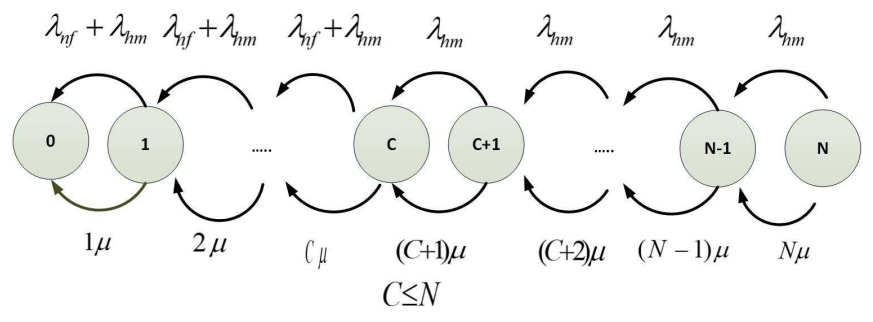

Fig. 4. State transitions of Markov chain model.

queue, where $1 / \mu$ is the mean service time. Similarly, the data packet length is formatted as arbitrary probability density function (PDF). The service time for arrival UEs is dedicated for processing handoff requests and $\mathrm{CAC}$ approval/reject registrations. Therefore, the arrival of handoff requests can be estimated using numerical predication that follows Poisson process. In such mechanism, the call arrival will be assigned a probability of $R$ for Request\#1 and $(1-R)$ to Request\#2. Requests \#1 and \#2 have Poisson arrival rates $R \lambda$ and $(1-R) \lambda$, respectively. For $n=1, \ldots, N$ number of arrival handoff requests during time interval $t$, the probability distribution function $R(n)$ is given as

$$
R(n)=\frac{\lambda t^{n}}{n} e^{-\lambda t}
$$

We also assume that $\lambda$ represents the traffic incurred by the system due to arrival UEs. This assumption is highlighted as the approximate value of data arrival process in dynamic systems. Therefore, we represent $\lambda_{h m}$ and $\lambda_{n s}$ as the average rate of handoff requests for subscribers moving from macrocell to small cell domain and the average request rate for subscribers moving from small cell to macrocell domain, respectively [9]. If the value of variable $C$ is less than $N$, the system will process requests originating from macrocell domain prior to any requests from originating from small cell domain. Where $N$ represents to the customers number in such system, and $C$ denotes to the total capacity in the system or number of waiting handoff requests. The handoff requests are modeled as state transitions using Markov chain procedure, as shown in Fig. 4. Markov chain procedure characterizes the proposed scheme to integrate policy making into macro and small cells to achieve QoS requirements. Predicting the traffic arrival is another factor that CAC considers to decide a handoff approval. In this way, the changes in traffic requirements for ongoing calls may significantly impact the available resource for any incoming calls subject to the overall available bandwidth. Therefore, the resource blocks are assigned in a sequential order and reserved for confirmed handoffs while allocating the unreserved blocks to the expected requests. An efficient CAC must simultaneously support an effective resources utilization and reasonable QoS to all arriving users. The analytical model considers the handoff mechanism presented in [8]. The value of $C$ for small cell domain can decrease the resource utilization when it is not updated regularly to reflect traffic changes at various domains. For a small cell with low rate of handoff requests, we can assume random values for $C$ and $N$. On the other hand, using random values of $C$ helps to simulate different status of resource optimization and handoff blocking probability. Therefore, the total arrival rate of handoff requests $\lambda_{j}$ is given as

$$
\lambda_{j}=\left\{\begin{array}{cc}
\lambda_{n s}+\lambda_{h m}, & \text { for } 0 \leq j<C \\
\lambda_{h m} & \text { for } C \leq j<N
\end{array}\right\} .
$$

Probability of blocking the calls originated in small cell is

$$
R_{B}^{\text {small }}=\sum_{i=C}^{N} \frac{\left(\lambda_{n s}+\lambda_{h m}\right)^{C} \cdot \lambda_{h m}^{i-C}}{i ! \mu_{m}^{i}} R(0) .
$$

The handoff policy for each access node is derived from the above equation using Markov process. For macrocell domain, the average call blocking probability is represented $R_{B, \text { Macro }}$ and the average call dropping probability is denoted $R_{D, M a c r o}$, that can be calculated as 


$$
\begin{gathered}
R_{H O}=\sum_{\forall s} N_{H O} * \pi \cdot N_{\text {calls }}(S), R_{B, \text { Macro }}=\sum_{i=C}^{C+N} R(i) \\
R_{B}^{M a c r o}=\sum_{i=K}^{C+N} \frac{\left(\lambda_{n s}+\lambda_{h m}\right)^{C} \cdot \lambda_{h m}^{i-C}}{i ! \mu_{m}^{i}} R(0) \\
R_{D}^{M a c r o}=R(C+N)=\frac{\left(\lambda_{n s}+\lambda_{h m}\right)^{C} \cdot \lambda_{h m}^{i-C}}{(C+N) i ! \mu_{m}^{i}} R(0)
\end{gathered}
$$

where $S$ is the probability of being in a certain state, $N_{H O}$ denotes the total number of macrocell and small cell handoffs that may occur at specific state. $N_{\text {calls }}$ represents the number of services belonging to a certain small or macrocell domain. Therefore, monitoring network performance can be performed by tracking the changes in state $S$ versus steady state probability values.

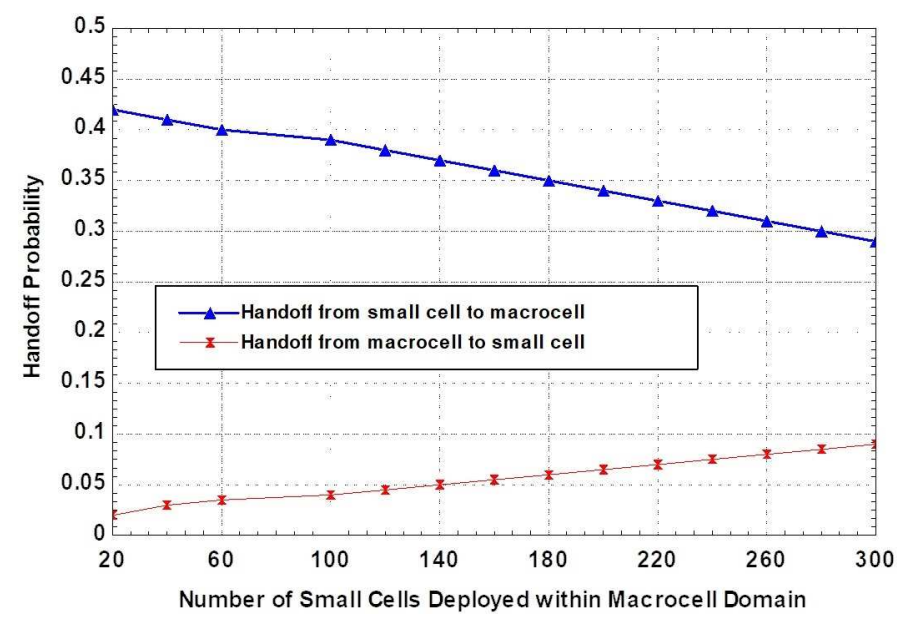

Fig. 5. Handoff probability for different number of small cells.

Fig. 5 shows handoff probability for the proposed CAC in particular state. Therefore, the handoff probability in the network is obtained by considering Markov states for ultradense networks. Increasing the number of small cells results in a significant increase in the probability of handoffs between macrocell and small cell domains. However, a reduced number of small cell in a macrocell domain does not necessarily increase handoff failure probability. Fig. 6 shows the performance fluctuations in data arrival rates due to changes in radio resource availability and number of small cells. The assumption is that proposed CAC mechanism is supported for all iterations and unlimited number of handoffs occur during each case scenario. It is clear that increasing the number of radio blocks along with increasing the number of small cells reduce the possibility of $R_{B, \text { Macro }}$ and results in higher number of delivered data. However, this performance modeling is based on UE moving from small cells to macrocell domain. Therefore, the data arrival rates are most likely to drop sharply when subscribers move from macrocell domain due to $R_{D, M a c r o}$. This also impacted significantly by the $N_{\text {calls }}(S)$ and session call changes. However, a reduced number of small cell in a macrocell domain does not necessarily increase handoff failure probability.

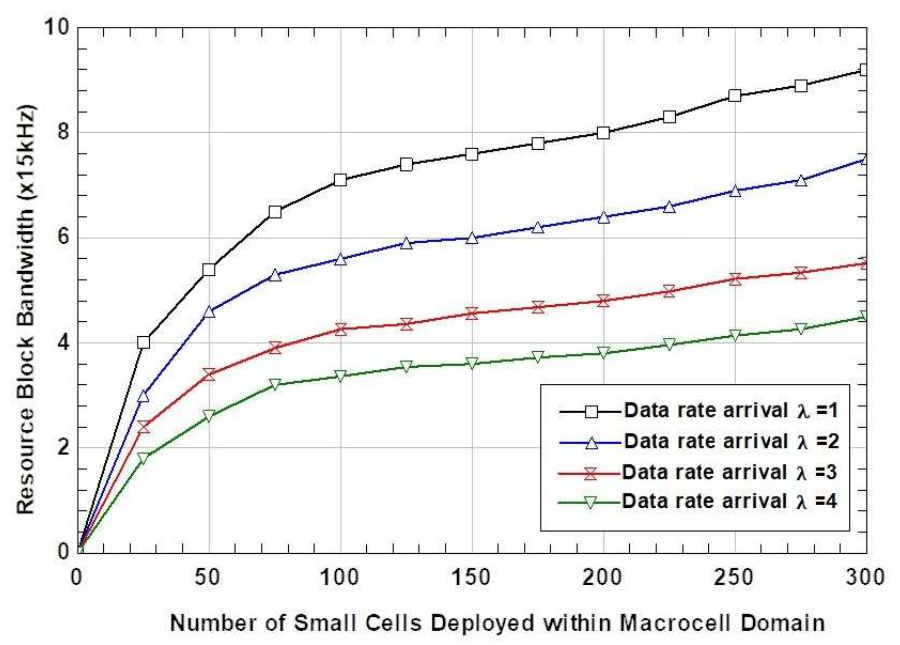

Fig. 6. Data arrival fluctuations for different CAC handoffs.

\section{CONCLUSION}

In this letter, an advance CAC scheme for 5G UDN was developed using Markov chain process. Regardless of the small cells density, results shown that multilayer end-to-end delay measurement and analysis are required to maintain secure system operation, while maintaining efficient utilization of available resources. It must be highlighted that the call admission control solutions specified in this letter guaranteed a low call blocking probability for various data traffic considering non-standalone $5 \mathrm{G}$ ultra-dense network overlaid small cells.

\section{REFERENCES}

[1] G. Brown, "Service-Oriented 5G Core Networks," White Paper, Huawei Technologies Co. Ltd., Feb. 2017.

[2] X. Ge, S. Tu, G. Mao, C. X. Wang, and T. Han, "5G Ultra-Dense Cellular Networks," IEEE Wireless Comms, vol. 23, no. 1, pp. 72-79, Feb 2016.

[3] A. Ksentini, T. Taleb, and K. B. Letaif, "QoE-Based Flow Admission Control in Small Cell Networks," IEEE Transactions on Wireless Communications, vol. 15, no. 4, pp. 2474-2483, April 2016.

[4] S. Y. Kim and C. H. Cho, "Call Blocking Probability and Effective Throughput for Call Admission Control of CoMP Joint Transmission," IEEE Transactions on Vehicular Technology, vol. 66, no. 1, pp. 622-634, Jan 2017.

[5] A. Al-Dulaimi, S. Al-Rubaye, Q. Ni, and E. Sousa, "5G Communications Race: Pursuit of More Capacity Triggers LTE in Unlicensed Band," IEEE Vehicular Technology Magazine, vol. 10, no. 1, pp. 43-51, March 2015.

[6] C. Yang, J. Li, Q. Ni, A. Anpalagan, and M. Guizani, "InterferenceAware Energy Efficiency Maximization in 5G Ultra-Dense Networks," IEEE Trans on Comms, vol. 65, no. 2, pp. 728-739, Feb 2017.

[7] M. R. Hossain, A. Z. M. A. H. Real, and A. Rahman, "Handover Management in Heterogeneous Network," in 2015 18th International Conference on Computer and Information Technology (ICCIT), Dec 2015, pp. 87-92.

[8] M. Z. Chowdhury, Y. M. Jang, and Z. J. Haas, "Call Admission Control Based on Adaptive Bandwidth Allocation for Wireless Networks," Journal of Comms and Nets, vol. 15, no. 1, pp. 15-24, Feb 2013.

[9] J. Huang and V. Krishnamurthy, "Cognitive Base Stations in LTE/3GPP Femtocells: A Correlated Equilibrium Game-Theoretic Approach," Communications, IEEE Transactions on, vol. 59, no. 12, pp. 3485-3493, December 2011. 